\title{
Vibration Analysis of Layered Composite Beam with Variable Section in Terms of Delamination and Orientation Angle in Analytical and Numerical Methods
}

\author{
G. Athihan ${ }^{a}$ And B. ERGene ${ }^{b, *}$ \\ ${ }^{a}$ Pamukkale University, Technology Faculty, Mechanical and Manufacturing Engineering Department, \\ 20160 Denizli, Turkey \\ ${ }^{b}$ Isparta Applied Sciences University, Technology Faculty, Mechanical and Manufacturing Engineering Department, \\ 32200 Isparta, Turkey

\begin{abstract}
In this study, vibration analysis of layered composite beams with variable cross sections and delaminations was investigated analytically and numerically. In the analytical part, Bernoulli beam theory was used and the effect of shear force on the beam was neglected. The layered composite beam consists of eight layers and contains delaminations with various sizes. In the numerical part of the study, ansys program package was used for finite element analysis. Layered composite beams with the same geometry were created in the finite element model. In the numerical part, the contact element was also defined between the delaminated parts. Furthermore, it has been shown numerically and analytically that the slope angle of the beam influences the vibration of the layered composite beam. Moreover, as the delamination between the beams increases, the vibration of the beam is observed to decrease. The layered composite beam is shown in graphical form in which the vibration can be changed without changing the geometry of the beam, showing that the vibration can be changed by changing the
\end{abstract} \\ fiber orientation angle.
}

DOI: 10.12693/APhysPolA.134.13

PACS/topics: vibration, composite, delamination, variable cross section

\section{Introduction}

Composite materials are widely used in almost every sector such as aero-space, automotive, military and textile, because of their unique property combination of low density and high strength. It is known that delamination damage causes the strength and rigidity of the layers to decrease. Delaminated layers generally exhibit new modes and frequencies of vibration depending on the number, location and size of the delaminations. In particular, delaminations reduce the natural frequency, which, if equal to the low operating frequency, causes resonance and causes damage to the structure. That is why, it is imperative that we can predict changes such as mode shapes in a dynamic environment.

In general, researchers have divided the regions of delamination into two parts, middle and edge. As shown in Fig. 1, the delamination width $b$, delamination length $a$ and beam length $L$ are given. They have studied the natural frequency changes by increasing the $a / L$ ratio of the beam or plate $(a / L=0.1, a / L=0.2, a / L=0.9)$. When the literature is examined, it is observed that the vibration analysis of the delaminated layered composite beam with variable section is very rare.

Jyothirmay and Ravikumar [1] carried out the vibration analysis of a rotating variable section composite

\footnotetext{
*corresponding author; e-mail: berkay_ergene@hotmail.com
}

beam. They have developed a composite beam model with variable cross section and compared it with the literature. In their analysis, they have studied the natural frequency of a rotating variable section composite beam, how it affects the taper ratio, fiber angle and rotation speed.
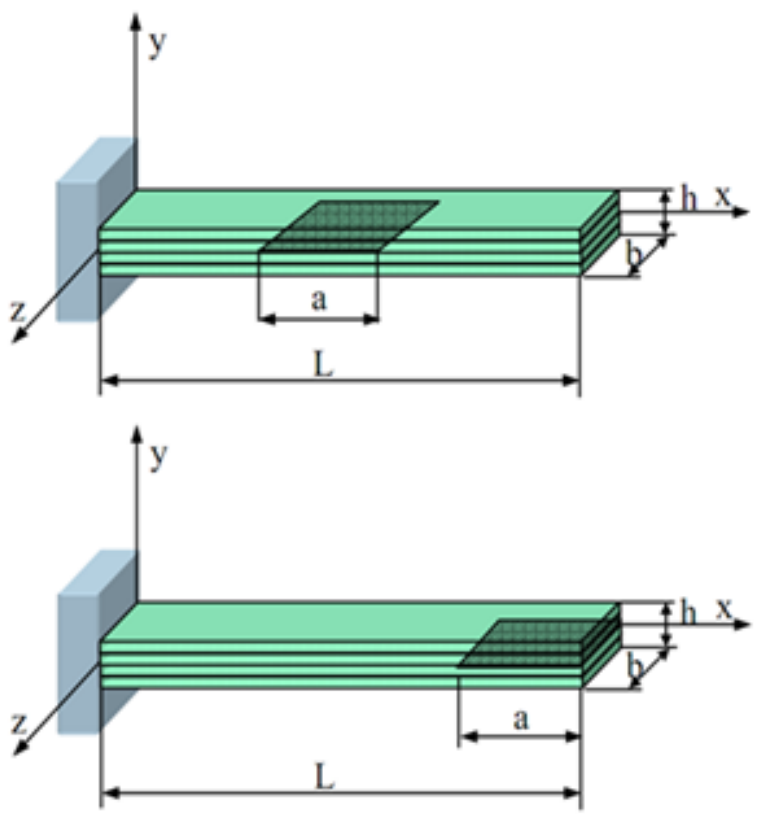

Fig. 1. Middle and edge delamination in beams. 
Arumugam and Rajamohan [2] investigated the free and forced reactions of the layered composite flake with varying cross-section with medium thickness delamination. Various parametric studies have been carried out to investigate the effects of variable section models, rotational speed, hole sizes and locations on the free vibration response of a rotating drilled variable section composite plate. As a result, it is concluded that the effects of delamination on the natural frequencies of plates with variable rotating speed are important.

Moreover, Hirwani and colleagues [3] examined the effect of delaminations on the free vibration behavior of curved layered composite panels in different geometries. It has also been observed that the position, position and size of the delamination, the geometric and material parameters as well as the laminated curved panel structures greatly influence the frequency response.

Furthermore, Balci and colleagues [4] studied the free vibrations of the layered composite beam in their work and solved the free vibration analysis of the finite element model of the beam where the strata were developed with different thickness conditions and different boundary conditions in the MATLAB program.

In their study, Torabi et al. [5] focused on the effect of vibration behavior using the experimental and the finite element methods, by applying various boundary conditions to cross-folded composite beams with delamination ratio of $0.20,0.10$ and 0.05 and investigated the importance of location and size of delamination.

In our study, vibration analysis of layered composite beams with variable cross sections and delaminations was analytically and numerically investigated. Bernoulli beam theory was used in analytical part and the effect of shear force on the beam was neglected. The layered composite beam contains eight layers and delaminations with various sizes.

For the numerical part of the work, ansys program package which uses the finite element method was used. Layered composite beams with the same geometry were created in the finite element model. In the numerical part, the contact element is also defined between the delaminated parts. Besides, it has been shown numerically and analytically that the slope angle of the beam influences the vibration of the layered composite beam. Moreover, when the delamination between the beams increases, the vibration of the beam is observed to decrease. As a result, it is possible to change the vibration of the composite beam by changing the fiber orientation angle without changing the geometry of the beam.

\section{Materials and methods}

\subsection{Analytical analysis of a layered composite beam}

Normal stress in layer number $j$ of a layered composite beam which can be seen in Fig. 2a is described by Eq. (1).

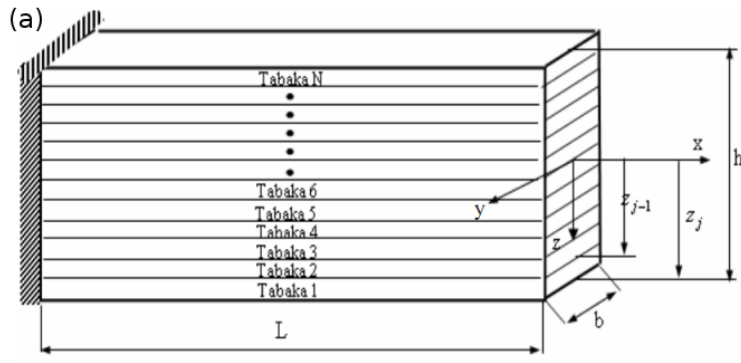

(b)

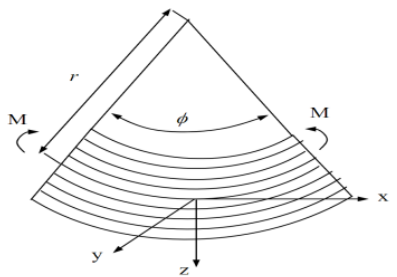

Fig. 2. (a) Layered composite beam. (b) Bending of the layered composite beam.

$$
\left(\sigma_{x}\right)_{j}=\left(E_{x}\right)_{j}\left(\varepsilon_{x}\right)_{j}
$$

According to the Bernoulli-Euler hypothesis (Fig. 2b), deformations at a certain distance from the neutral surface are given by Eq. (2).

$$
\varepsilon_{x}=\frac{(r+z) \phi+r \phi}{r \phi}=\frac{z}{r} .
$$

Here $r$ is the radius of curvature.

Relationship between the normal stress and the bending moment is

$$
M=\frac{2 b}{3 r} \sum_{j=1}^{N}\left(E_{x}\right)_{j}\left(z_{j}^{3}-z_{j-1}^{3}\right),
$$

where $h$ is the height of the beam, $b$ is the width of the beam, $N$ is the number of layers, and $z_{j}$ is the distance between the outer surface of the $j_{\text {th }}$ layer and the neutral surface.

The relationship between the bending moment and the radius of curvature can be written as follows [6];

$$
\begin{aligned}
& M=\frac{E_{f} I}{r}=E_{f} I \frac{\mathrm{d}^{2} w}{\mathrm{~d} x^{2}}, \\
& E_{f}=\frac{8}{h^{3}} \sum_{j=1}^{N}\left(E_{x}\right)_{j}\left(z_{j}^{3}-z_{j-1}^{3}\right), \\
& E_{z d}=\frac{1}{z} \sum_{i=1}^{m} E_{f i} z_{i}, \\
& E_{d}=\left(E_{z d}-E_{f}\right) \frac{A_{d}}{A_{t}}+E_{f} .
\end{aligned}
$$

In these equations, $z_{i}$ is the thickness of the $i$ th layer, $z$ is the thickness of the beam, $m$ is the number of delaminations, $E_{f}$ is the effective modulus of elasticity, $E_{z d}$ is the modulus of elasticity in the delaminated layer, $E_{d}$ is the modulus of elasticity of the delamination beam. 
Relation for the static load moment for a beam subjected to load is;

$$
\frac{\mathrm{d}^{2} M}{\mathrm{~d} x^{2}}=\frac{\mathrm{d} V}{\mathrm{~d} x}=p(x) .
$$

It is transformed into

$$
\frac{\mathrm{d}^{2}}{\mathrm{~d} x^{2}}\left(E_{f} I_{d} \frac{\mathrm{d}^{2} w}{\mathrm{~d} x^{2}}\right)=p(x) .
$$

The d'Alembert principle is used for the dynamic load. Mass and the acceleration must be added to the above equation. Thus we obtain;

$\frac{\mathrm{d}^{2}}{\mathrm{~d} x^{2}}\left(E_{f} I_{d} \frac{\mathrm{d}^{2}(w, t)}{\mathrm{d} x^{2}}\right)=p(x, t)-\rho_{\mathrm{m}} A \frac{\mathrm{d}^{2} w(x, t)}{\mathrm{d} t^{2}}$.

Here $\rho_{\mathrm{m}}$ is the density of material, $A$ is cross-sectional area, and $I_{d}$ is the moment of inertia of the beam with variable cross section.

The natural frequency of the beam depends on the material properties and geometry, not on the applied force. Thus $p(x, t)$ can be assumed to be zero and the equation becomes

$$
\frac{\mathrm{d}^{2}}{\mathrm{~d} x^{2}}\left(E_{f} I_{d} \frac{\mathrm{d}^{2}(w, t)}{\mathrm{d} x^{2}}\right)+\rho_{\mathrm{m}} A \frac{\mathrm{d}^{2} w(x, t)}{\mathrm{d} t^{2}}=0 .
$$

This is a homogeneous equation and the coordinates- and time-dependent harmonic components of the vertical direction that provides boundary conditions is

$$
w(x, t)=\sum_{n=1}^{\infty} A_{n} \sin \frac{n \pi x}{L} \cos \omega_{n} t,
$$

where $A_{n}$ is the amplitude and $n$ is the index of a natural frequency. Equation (12) is solved for the Eq. (11), $\omega_{n}$ is found for each $n$. $\omega_{n}$ is the frequency value for the variable beam section.

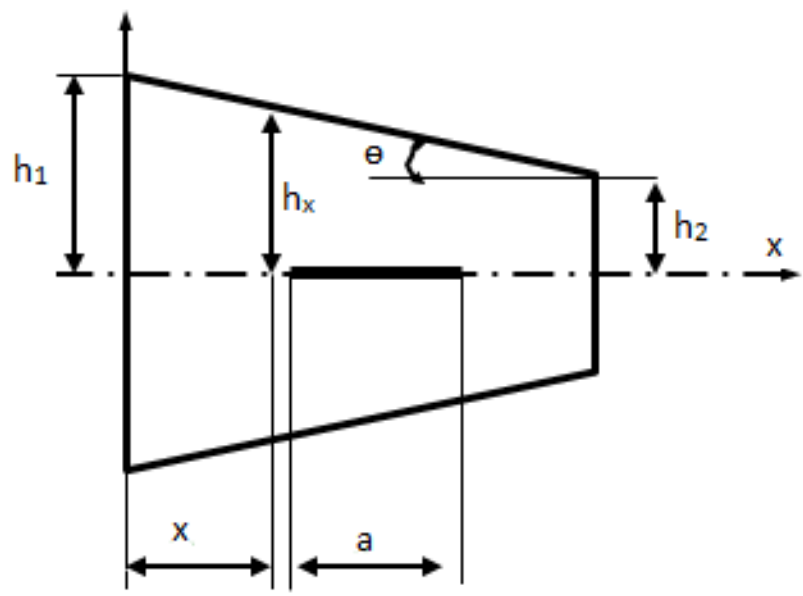

Fig. 3. Variable section with delamination length $a$.

Since the beam has a variable cross section (Fig. 3), it is defined by function $f(x)$ with variable intercept [7]. $\theta$ is conic angle, $\beta$ is variable section parameter, $h_{x}$ is half thickness depending on $x, h_{1}$ is maximum half thickness, $h_{2}$ is minimum half thickness.

$$
\begin{aligned}
& h_{x}=h_{1}[1-\beta f(x)], \\
& \beta=\left(1-\frac{h_{2}}{h_{1}}\right), \\
& f(x)=\frac{x}{L} .
\end{aligned}
$$

The moment of inertia $I_{d}$ is calculated according to the selected function.

\subsection{Numerical analysis of layered composite beam}

First of all, tensile tests of layered composite beams were carried out to determine their elasticity modulus and the obtained geometrical and material properties of the girder are given in Table I.

There are many computer programs that use the finite element method and one of the most popular is ANSYS [8]. In this study, the type of element to be used was first determined. The element to be analyzed must have vibrational, layered, composite properties. Shell99 was chosen as the element that provides these properties. Shell99 can be used for layered structural shell models and allows to work with up to 250 plates. This element rotates in $x, y, z$ directions and is fixed to six degrees of freedom. It is used in orthotropic (composite) materials. When mesh is made in Shell99 (Fig. 4) the element can be divided into cubic or triangular element pieces.

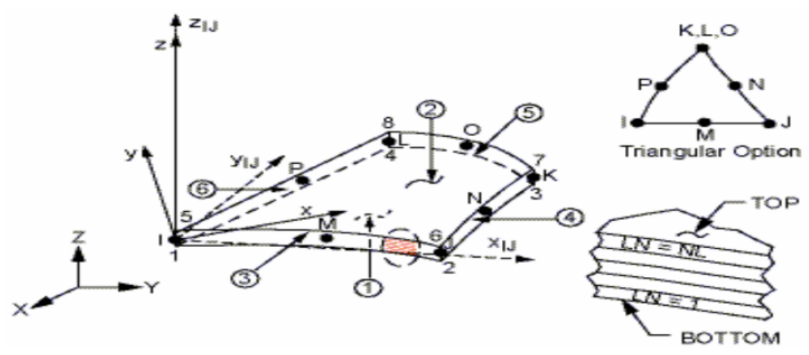

Fig. 4. Geometry of shell element.

TABLE I

Material properties and dimensions of the laminated composite beam.

\begin{tabular}{l|c|c}
\hline \hline \multicolumn{1}{c|}{ Properties } & Symbol & Value \\
\hline Longitudinal elasticity modulus & $E_{1}[\mathrm{MPa}]$ & 44150 \\
Transverse elasticity modulus & $E_{2}[\mathrm{MPa}]$ & 12300 \\
Shear modulus & $G_{12}[\mathrm{MPa}]$ & 4096 \\
Poisson's ratio & $\vartheta_{12}$ & 0.2 \\
Length & $L[\mathrm{~mm}]$ & 400 \\
Height & $h[\mathrm{~mm}]$ & 3 \\
Width & $b[\mathrm{~mm}]$ & 20 \\
Delamination length & $\mathrm{a}[\mathrm{mm}]$ & 40
\end{tabular}

It can be seen from Fig. 5a that the laminated composite beams which have middle delamination were modelled by using four areas. First area A1 was glued to both areas A2 and A3, but interface of A2 and A3 is not glued 
in the beams with the middle delamination. In the same way, area A4 was glued to areas A2 and A3. Therefore, delamination has been formed between areas A2 and A3, as seen in Fig. 5a.

Double areas occur at the same coordinates of the interfacial areas after areas have been transformed into meshes. In conclusion, the composite beams were formed as contact elements between areas A2 and A3, so that the areas with the delamination could be moved together.

The contact element was selected as Contact 174 element type, as can be seen in Fig. 5b. Thus, the laminated composite beams had middle delamination. After the mesh generation process, a delaminated composite beam with delamination ratio $a / L=0$ had 1000 elements and 2976 nodes. By increasing delamination ratios, the number of elements and nodes of the beams increased as well.

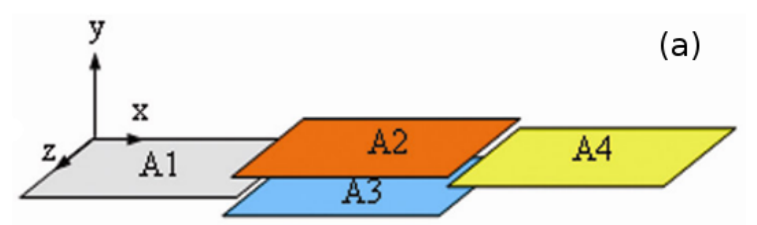

(b)

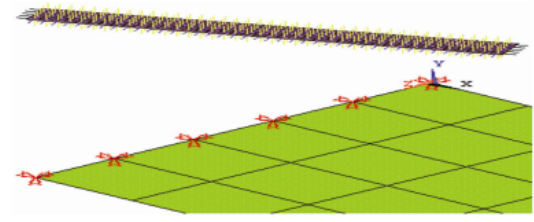

Fig. 5. (a) Layered composite beam with middle delamination. (b) View of a laminated composite beam with contact element and boundary conditions and mesh shape.

\section{Results and discussion}

In this study, vibrational behavior of laminated composite beams having clamped-free boundary condition, single-middle delamination and various stacking sequences are investigated analytically and numerically. The laminated composite beams are modeled as single middle delamination by using ANSYS, a commercial software used in the numerical solution of problems by FEM.

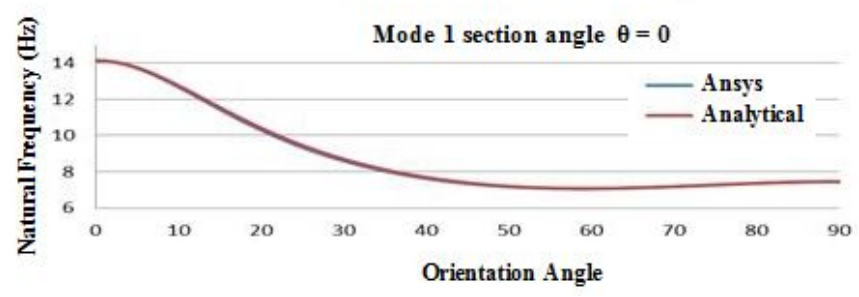

Fig. 6. Analytical and numerical change of natural frequency of layered composite beam according to layer angle (mode 1 ).
When the cross-section angle of the layered composite beam $\theta=0$, the natural frequency of the beam angle is affected. In Fig. 6, the direction of the orientation of the stratified composite beam reaches the maximum value at zero and the frequency falls rapidly. The decrease in this frequency lasted to $58^{\circ}$. The lowest frequency value was found at this angle. The natural frequency of the stratified composite beam appears to increase somewhat from $59^{\circ}$ up to $90^{\circ}$. In addition, both analytical and numerical results are close to each other. When the crosssection angle of the layered composite beam is $\theta=0$, the natural frequency of the beam angle is affected.

In Fig. 7 the direction of orientation of the layered composite beam appears to reach the highest value for all modes at zero. The modal number increased the natural frequency of the composite beam with residual layer. The sectional angle of the layered composite beam is affected by the natural frequency of the beam angle when $\theta=0$, $\theta=0.1$ and $\theta=0.2$.

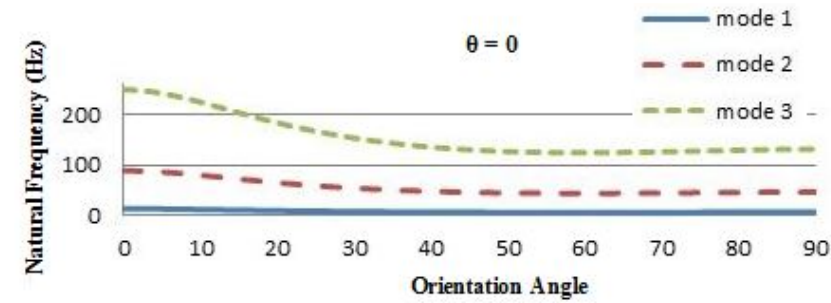

Fig. 7. Variation of natural frequency of layered composite beam for various modes with respect to layer angle.

In Fig. 8, the direction of orientation of the layered composite beam reaches the highest value at zero and the frequency drops rapidly. The reduction of this frequency lasted between $0^{\circ}$ and $61^{\circ}$ and the lowest frequency value was found at these value. The natural frequency of the layered composite beam increases from $61^{\circ}$ to $90^{\circ}$. The cross-section angle of the layered composite beam is $\theta=0.1$, whereas the natural frequency of the beam angle is affected.

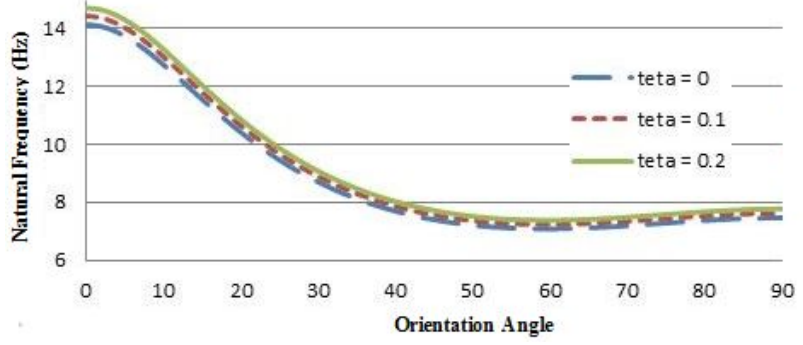

Fig. 8. Change of natural frequency of layered composite beam with respect to layer angle (mode 1 ).

In Fig. 9 the direction of orientation of the stratified composite beam reaches the maximum value at zero point, and the frequency rapidly falls. The reduction of this frequency lasted between $0^{\circ}$ and $61^{\circ}$ and the lowest 
frequency value was found at these values. The natural frequency of the layered composite beam increases from $61^{\circ}$ to $90^{\circ}$. The residual frequency values are reduced with increase of $a / L$ for all orientation angles.

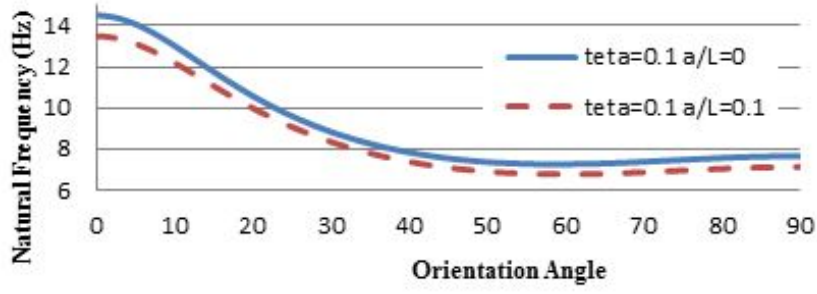

Fig. 9. Change of the natural frequency of the layered composite beam with respect to the layer angle when $a / L=0.1$ (mode 1$)$.

\section{Conclusions}

In this study, the natural frequencies of composite beams with clamped-free boundary condition and with single-middle delamination are investigated analytically and numerically. In the analytical solution, Bernoulli beam theory is used. In numerical solution, ANSYS finite element code is used and when the beams are modeled, the contact elements are defined. The largest frequency values of the layered composite beam are found at $0^{\circ}$. Frequency values from $0^{\circ}$ to about $59^{\circ}-60^{\circ}$ have been observed to decrease. A slight increase in the frequency of the layered composite beam was observed above 60 degrees.

When the number of modes of the beam increases, it is seen that the frequencies increase. Furthermore, increasing the cross-section angle $\theta$ of the layered composite beam increases the natural frequency.
It has been observed that the natural frequency can be changed by changing the layer angle of the layered composite beam. This shows us that we can change the natural frequency of the beam without changing the geometry of the beam. In addition, as the $a / L$ value increases, the frequency values decrease for all orientation angles.

\section{Acknowledgments}

Authors would like to thank Pamukkale University Scientific Research Council for supporting this study under project contract No. 2012BSP010.

\section{References}

[1] U. Jyothirmayi, P. Ravikumar, International Research Journal of Engineering and Technology 3(8), 999 (2016).

[2] A.B. Arumugam, V. Rajamohan, Aerospace Science and Technology 60, 172 (2017).

[3] C.K. Hirwani, TR.K. Patil, S.K. Panda, S.S. Mahapatra, S.K. Mandal, L.S. Srivastava, M.K. Buragohain, Aerospace Science and Technology 54, 353 (2016).

[4] M. Balcı, M.O. Nalbant, E. Kara, Ö. Gündoğdu, International Journal of Automotive and Mechanical Engineering 9, 1734 (2014).

[5] K. Torabi, M. Shariati-Nia, M.Heidari-Rarani, International Journal of Mechanical Sciences 115-116, 1 (2016).

[6] R.F. Gibson, Principles of composite material mechanics, McGraw-Hill, New York 1994.

[7] S.R Ramalinggeswara, N. Ganesan, Journal of Sound and Vibration 187(5), 737 (1995).

[8] Ansys 10.0 User's Manual, 2005. 